\title{
La Alfabetización Matemática y su Relación con el Intercambio Comercial, la Escolaridad Elemental y el Trabajo
}

\section{Mathematical Literacy and its Development through Elementary-education Programs and Occupations that Do not Require much Schooling*}

Alicia Avila**

\begin{abstract}
Resumen
En este artículo abordo algunos aspectos de la adquisición del conocimiento y las herramientas matemáticas elementales en diferentes contextos: la educación básica de personas jóvenes y adultas, algunas actividades laborales que no requieren una escolaridad importante, y la experiencia de intercambio comercial que se desarrolla en la cotidianeidad. Mi intención es contribuir a la reflexión sobre los aportes de la educación básica, la experiencia cotidiana y el trabajo a la alfabetización matemática de las personas. Para ilustrar mis afirmaciones, tomo como centro la noción de proporcionalidad. Esta noción cruza la educación básica, es fundamento de la matemática más avanzada y, también, es instrumento de trabajo e interacción en distintos ámbitos de la actividad social. Adelanto que, en México, el sistema educativo para jóvenes y adultos incumple en buena medida un compromiso fundamental: lograr que las personas se apropien de herramientas simbólicas que potencien su pensamiento y su forma de vinculación con el mundo. En cambio, algunas actividades laborales y comerciales contribuyen al desarrollo de conocimientos y habilidades útiles para resolver problemas matemáticos que el propio contexto plantea. Este tipo de aprendizaje, no debe empero olvidarse, es un aprendizaje situado cuyos límites conviene también señalar.
\end{abstract}

\footnotetext{
* Agradezco a Hugo Espinosa Pérez el cuidado con que revisó y comentó la primera versión de este artículo.

** Doctora en Pedagogía por la Universidad Nacional Autónoma de México (UNAM). Investigadora Titular de la Universidad Pedagógica Nacional, México D.F., México. Dirección del Correo: Guty Cárdenas 121-B, Col. Guadalupe Inn., C.P. 01020, México D.F., México. E-mail: aliavi@prodigy.net.mx
} 
Palabras clave: Alfabetización Matemática. Educación Básica. Jóvenes y Adultos. Proporcionalidad. Procesos Cognitivos. Estrategias de Solución.

\begin{abstract}
My goal is to contribute to the discussion of how elementary education and labor activities contribute to the mathematical literacy of people. I examine some aspects of how youths and adults acquire mathematical knowledge and tools, both through elementary-education programs and through engaging in occupations that do not require much schooling. I focus on the concept of proportionality because it cuts across elementary education, it serves as a base for more advanced mathematics, and it is an asset for work and for interaction in different fields of social activity. I explain how the Mexican educational system fails to accomplish one of its fundamental obligations: to ensure that the people it serves obtain the symbolic tools that are necessary for enhancing their mathematical thinking, and their ways of relating to the world. I also explain how some occupational and commercial activities contribute to people's development of mathematical knowledge and skills that are useful for dealing with problems that emerge in specific contexts. However, I argue that it is important to keep in mind that the latter kind of learning is strongly situated and has limitations worth pointing out.
\end{abstract}

Keywords: Mathematical Literacy. Elemental Education. Youths and Adults. Proportions, Cognitive Process. Solution Strategies.

\title{
1 Antecedentes
}

El contenido de este artículo se sustenta en una indagación que realicé con un grupo de colegas en dos entidades federativas de México: el estado de Aguascalientes y el Distrito Federal (AVILA et al.; 2008). En dicha investigación se abordaron temas como la proporcionalidad, el cálculo de áreas, el cálculo aritmético y la lectura de tablas de información y gráficas con jóvenes y adultos que no habían concluido su educación básica en la infancia, y que lo hacían posteriormente orientados por distintos objetivos, todos asociados al deseo de superación ${ }^{1}$. Aquí abordaré sólo la proporcionalidad, tema que ilustra bien sobre lo que ocurre con otros contenidos matemáticos también incluidos en el currículum de educación básica de jóvenes y adultos. Tangencialmente, referiré al cálculo aritmético y al cálculo de áreas en un contexto de construcción.

\footnotetext{
${ }^{1}$ Respecto de los temas mencionados pueden verse los trabajos de Eudave (2009) y Estrada y Avila (2009), que abordan respectivamente los temas de lectura e interpretación de gráficas y cuadros con información numérica, así como la resolución de problemas de área.
} 
El objetivo al realizar la investigación antes mencionada fue responder a las siguientes preguntas:

- ¿Se adquieren en la Educación Básica de Jóvenes y Adultos (EBPJA) las herramientas que constituyen los procedimientos expertos y generales propios de la matemática escrita convencional?

- ¿Qué aportan otras fuentes de experiencia, como son el intercambio comercial y la actividad laboral a la alfabetización matemática de las personas?

- ¿Las herramientas escolares se utilizan para resolver los problemas que las personas enfrentan cotidianamente?

- ¿La eventual posesión de dichas herramientas mejora la capacidad de resolver problemas vinculados a la experiencia?

Estas cuestiones refieren a la comprensión y uso funcional del conocimiento matemático. Es decir, nos remiten a lo que puede llamarse alfabetización matemática, si ésta se entiende como: "La capacidad de identificar información cuantitativa, valorarla críticamente, reflexionar sobre ella y a partir de ella, y aplicarla al enfrentar actividades y problemas del ámbito social, laboral y personal"2 (EUDAVE, 2010).

Las habilidades de este tipo son relevantes como objeto de investigación, ya que tradicionalmente han sido consideradas como un área básica de destrezas. Una revisión somera de las tareas o problemas comunes en la vida de muchos adultos urbanos es suficiente para revelar la diversidad y riqueza de situaciones donde estos tipos de habilidades se necesitan: la compra de alimentos, el llenado de formatos bancarios y fiscales, el manejo de las finanzas personales, el uso de mapas y planos, las mediciones necesarias para arreglar o reparar una casa, la lectura de noticias que incluyen datos estadísticos y resultados de encuestas, la lectura de informaciones médicas, la ayuda a los niños con los trabajos escolares, entre otras (GAL, 1993; VERGNAUD, 2002). ${ }^{3}$

\footnotetext{
${ }^{2}$ Esta definición es una revisión de la elaborada por Eudave, en la invitación al Coloquio Alfabetización Académica, Informativa y Numérico-estadística en universitarios, realizado en la Universidad Autónoma de Aguascalientes en noviembre de 2010, evento para el cual elaboré la primera versión de este escrito.

${ }^{3}$ Sé que actualmente la noción de competencia - y por tanto la de competencia matemática - ha tomado un lugar privilegiado, si no en las discusiones, sí en el discurso sobre la enseñanza y el aprendizaje en general y de las matemáticas en particular. En mi opinión, este concepto es aún motivo de debates y cuestionamientos, por lo que prefiero utilizar el término de alfabetización matemática tal como recién se definió.
} 


\section{Metodología del estudio}

\section{1 ¿Por qué la proporcionalidad?}

La proporcionalidad es un contenido importante en el currículum de educación básica de jóvenes y adultos (EBJA). Es, también, un saber matemático útil en muchos trabajos y oficios; por ejemplo el de comerciante en sus distintas modalidades, o el de agricultor (SOTO; ROUCHE, 1995). Las personas que trabajan en el ámbito de la construcción, como los pintores, los marmoleros y los maestros albañiles también la utilizan al realizar algunas de sus tareas (DE AGÜERO, 2006; AVILA et al., 2008; CARRAHER; CARRAHER; SCHLIEMANN, 1991). Todos hacemos uso de la proporcionalidad cuando calculamos costos; por ejemplo, el de 10 refrescos si la caja de 24 vale $\$ 252$ y se mantiene el precio al comprar por unidad, o cuando calculamos el cargo por el adeudo en nuestra tarjeta bancaria si el interés es, por ejemplo, de $4 \%$ mensual. Esta dualidad de la proporcionalidad - como herramienta de utilidad práctica y como contenido escolar básico - nos llevó a incluirla en nuestra investigación, bajo el interés de incorporar problemas vinculados a la vez con temáticas del currículum de educación básica y con la experiencia cotidiana de los entrevistados.

\subsection{Las personas participantes en la investigación}

Para responder las preguntas que nos planteamos, se entrevistó y se planteó un conjunto de problemas matemáticos con las características recién señaladas, a 28 jóvenes y adultos del medio urbano o rural, que asistían al servicio de educación básica (primaria o secundaria) en dos entidades de la república mexicana: el estado de Aguascalientes y la ciudad de México. Los problemas fueron planteados por escrito y de manera individual. Si las personas lo necesitaban, se les ayudaba a leerlos o se leía para ellas cada uno de los problemas hasta que, suponíamos, se había logrado su comprensión. Los entrevistados eran comerciantes, mozos, o trabajadores de la construcción; las mujeres, en su mayoría, eran empleadas domésticas, pero también había trabajadoras de la industria maquiladora, algunas dedicadas al comercio, o eran amas de casa. Entre los jóvenes, la mayoría se dedicaba sólo a estudiar. El rango de edades iba de 15 a 62 años. 


\subsection{Los dos tipos de conocimiento}

Tanto en la vida cotidiana como en la educación básica, la proporcionalidad ocupa un lugar importante. Pero el conocimiento que implica en un caso se desarrolla con las reglas del cálculo oral (el que no se escribe) y, en el otro, con las reglas de la matemática escrita, cuyos algoritmos y procedimientos de solución se trasmiten en las escuelas.

Conforme a la perspectiva que he ido construyendo a lo largo de mi actividad de indagación, postulo que la adquisición significativa de simbolizaciones y procedimientos escritos (escolares) potencia la capacidad de resolver problemas que se desarrolla en la vida, porque constituyen herramientas simbólicas que permiten desligar al pensamiento del aquí y el ahora propio de la matemática cotidiana. Como han señalado Carraher y sus colegas (CARRAHER; CARRAHER; SCHLIEMANN, 1991), ambos elementos actúan como amplificadores de la capacidad de calcular.

Mi interés, con base en esta postura, fue conocer qué conocimientos y herramientas aporta la educación básica para jóvenes y adultos (EBPJA) a las personas, y qué tanto éstas contribuyen al desarrollo de su capacidad de solucionar problemas de proporcionalidad, independientemente de la actividad laboral que realizan. Y, aunque este no fue el foco inicial de la indagación, el trabajo en el terreno permitió colectar datos sobre los aportes dela experiencia laboral y la actividad comercial al desarrollo de destrezas matemáticas.

\section{4 ¿Por qué un contexto comercial?}

Escogí el contexto comercial para plantear la situación sobre proporcionalidad por ser el que según otros estudios (por ejemplo, MARIÑO, 1983; 1997; AVILA, 1990), es familiar a la gran mayoría de las personas jóvenes y adultas, quienes, en general, desarrollan alguna actividad económica o laboral vinculada al manejo del dinero y al pago o cobro de servicios y mercancías. Esta afirmación es válida incluso para el caso de los campesinos y grupos indígenas habitantes de pequeñas comunidades, quienes, en general, incluyen como corolario de su actividad agrícola, la venta de sus productos, en grandes o pequeñas cantidades (SOTO; ROUCHE, 1995; AVILA et al., 2010). 


\subsection{La situación planteada}

Se propuso una situación en la que se requería resolver seis problemas de diversa dificultad consistentes en calcular el precio de varios trozos de ate (dulce) de membrillo de distinto peso. Nuestro supuesto era que la mayoría de las personas habrían enfrentado en su vida situaciones análogas a ésta (véase Figura 1).

En la tienda de don Fernando empacan trozos de ate de membrillo ${ }^{4}$ de distinto peso. Ya pusieron la etiqueta a un paquete. Fíjese en el precio y complete las etiquetas que faltan.

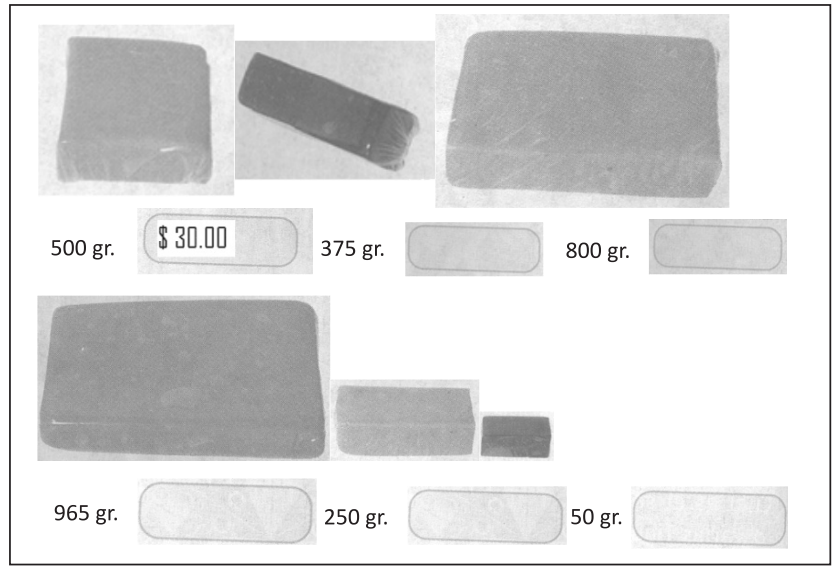

Figura 1 - Situación de proporcionalidad planteada durante la investigación

Los problemas planteados son del tipo valor faltante: se conocen tres datos y se desconoce el cuarto dato de la proporción. Para la resolución de todos ellos se tenía como referente la relación siguiente: 500 gramos de cajeta cuestan \$30.00, que implica la razón 30/500 entre el precio y el peso.

\subsection{Posibles estrategias de solución}

Todos los problemas planteados pueden resolverse fácilmente, utilizando el precio del gramo o la regla de tres. Pero hace tiempo sabemos - con base en las indagaciones realizadas con niños y jóvenes escolarizados - que este tipo de problemas también puede solucionarse sin recurrir al valor de la unidad y sin

\footnotetext{
${ }^{4}$ El ate de membrillo es un dulce típico de algunas regiones de México.
} 
utilizar la relación funcional entre las cantidades. Este hecho lo han destacado recientemente Fernández, Llinares y Valls (2011), quienes sintetizan en cuatro categorías los distintos enfoques y estrategias para resolver problemas que implican razonamiento proporcional:

- Enfoque funcional. Basado en el uso de la razón externa.

- Reducción a la unidad (caso particular del enfoque funcional).

- Enfoque escalar. Basado en el uso de razones internas.

- Enfoque constructivo. Basado en el uso de la propiedad $f(a+b)=f(a)+f(b)$.

En el enfoque escalar, se trabaja con las relaciones al interior de un mismo tipo de cantidades (por ejemplo, los pesos de los distintos trozos de ate en el problema planteado) y luego se reproducen esas relaciones en el otro tipo de cantidades (los distintos precios solicitados).

Esta forma de calcular, basada en las relaciones escalares, permite resolver ciertos problemas de proporcionalidad sin utilizar el enfoque funcional al cual se vinculan los procedimientos típicamente escolares: el cálculo del valor unitario (el costo del gramo en nuestro ejemplo), o la regla de tres correspondiente.

\section{Capacidad de resolver los problemas de proporcionalidad e incorporación de herramientas escolares ${ }^{5}$}

Para el análisis de las respuestas y estrategias de solución, consideré la corrección de las respuestas, así como su grado de exactitud, porque son indicadores de: a) la destreza con que se enfrentan los problemas; b) el grado de incorporación de las simbolizaciones y procedimientos escolares en las formas de resolver los problemas.

La destreza observada en nuestros entrevistados varió notablemente, dependiendo de los problemas. La gran mayoría (más de 80\%) dio respuestas correctas y exactas en los problemas más sencillos y posibles de resolver con la estrategia "duplicar o sacar mitad al dato conocido", por ejemplo al calcular el precio del kilo: Nomás veo que el kilo es el doble del medio (kilo, 500 gr) y ya saco la cuenta: son $\$ 60$ [por el kilo] ${ }^{6}$.

\footnotetext{
${ }^{5}$ Una versión inicial de los argumentos planteados en esta parte del artículo los expuse en otro trabajo (AVILA, 2009); una relectura de los datos me permitió profundizar el análisis y hacer algunas modificaciones en la interpretación de los mismos.

${ }^{6}$ Para una perspectiva sobre el conjunto de las respuestas en términos de las dos categorías mencionadas, puede verse al tabla anexa al final del escrito.
} 
En cambio, en los problemas donde la estrategia de duplicar o sacar mitad es insuficiente y se requieren procedimientos espontáneos más elaborados - haciendo uso de la propiedad $f(a+b)=f(a)+f(b)-$ o los procedimientos escolares, que permiten resolver con exactitud cualquier caso, la mayoría de las personas deja de ser competente para obtener las soluciones. Efectivamente, en ninguno de los problemas que obligan al uso de estos procedimientos se alcanzó un $50 \%$ de respuestas correctas y exactas. La mayoría de los entrevistados los resolvió simplemente mediante aproximaciones (ver tabla al final del escrito).

¿Cuál es la importancia de este dato? Que resolver con exactitud todas las preguntas planteadas implicaba calcular el valor unitario o la regla de tres. Nuestro supuesto al iniciar la indagación era que a mayor escolaridad habría un uso más frecuente de estas estrategias y que, utilizándolas, las personas estarían en posibilidad de resolver los problemas donde las estrategias no escolares dejan de ser efectivas. Pero esta hipótesis no encontró sustento empírico. Las personas - aun las que estaban por concluir la secundaria - no recurrieron a las estrategias escolares escritas ni en los problemas en que éstas se hacen indispensables para calcular el precio exacto. En lugar de recurrir a ellos, se utilizaron estrategias propias del cálculo oral, basadas en el enfoque escalar, ocasionalmente acompañadas de anotaciones personales para apoyar el cálculo.

Estas estrategias pueden ser simples y económicas en algunos casos pero, como veremos adelante, también pueden resultar engorrosas y, como se ha señalado respecto de los esquemas de acción (VERGNAUD, 2002), no necesariamente son efectivas en el sentido de alcanzar la solución buscada a partir de un número finito y sistemático de pasos, efectividad que sí aseguran los algoritmos convencionales.

\section{Estrategias utilizadas para resolver los problemas de proporcionalidad}

\subsection{Sin estrategias para enfrentar los problemas}

Algunas mujeres asistentes al tramo final de la primaria, y dedicadas a labores domésticas, hicieron intentos por resolver los problemas planteados, pero terminaron por no responder ninguno. Las razones de no hacerlo se expresaron de la siguiente manera:

En las tiendas tienen sus básculas y uno ni le entiende a esas cosas (mujer del medio rural). 
Es que yo no hago las cuentas, voy al súper y ahí ya vienen los precios (mujer habitante de la ciudad de México, empleada doméstica).

Se ven aquí dos cuestiones. La primera es que la experiencia de vida no ha obligado a estas mujeres a resolver problemas de pesos-precios, porque otras personas calculan o pesan para ellas. Esto se ha traducido en un escaso desarrollo de las habilidades necesarias para resolver problemas de proporcionalidad. En tales circunstancias, la responsabilidad de calcular bien los precios se deja a los vendedores o a las máquinas. La otra cuestión que es visible, está relacionada con el papel de la tecnología. Es evidente que la tecnología utilizada para pesar y cobrar los productos en los almacenes modernos actúa en una doble vía en cuanto al desarrollo de las habilidades de cálculo. Por una parte facilita e incorpora efectividad en la tarea cotidiana de hacer cuentas. Por otra parte, al volverlas innecesarias, inhibe el desarrollo de habilidades para calcular. Pero en el caso de estas mujeres, llama la atención que tampoco se han incorporado las estrategias escolares para resolver los problemas, aunque estén por concluir su educación primaria.

\subsection{Enfoque escalar: duplicación y mitad del valor conocido}

En párrafos anteriores señalé la duplicación y la obtención de la mitad del valor conocido como la estrategia utilizada para resolver problemas sencillos de proporcionalidad. Si se sabe que 500 gramos cuestan $\$ 30.00$ entonces, por isomorfismo, como el kilo es el doble de 500 (1000 gr), el costo será el doble de \$30 (\$60) y, 250, al ser la mitad de 500gr costarán sólo \$15, que es la mitad de $\$ 30$. Esta duplicación o subdivisión puede reiterarse cuantas veces se necesite para obtener el valor buscado. Por ejemplo para calcular lo que costarían $125 \mathrm{gr}$, se haría necesario obtener una mitad más: la mitad de \$15 (\$7.50).

\subsection{Uso de la propiedad $f(a+b)=f(a)+f(b)$ : sumas $(y$ restas $)$ de duplicaciones (o mitades) sucesivas}

Se identificaron estrategias más elaboradas que la simple duplicación o división por dos, basadas también en relaciones escalares, a saber: sumas o restas de los valores conocidos y/o de las mitades y dobles sucesivos obtenidos. Según esta forma de operar, la estrategia para calcular el precio de 375 gramos consiste en obtener dos veces sucesivamente la mitad de 500 y sumar los valores resultantes $(250+125)$, para después hacer lo mismo con los valores correspondientes en los precios $(15+7.50)$ (Figura 2). 


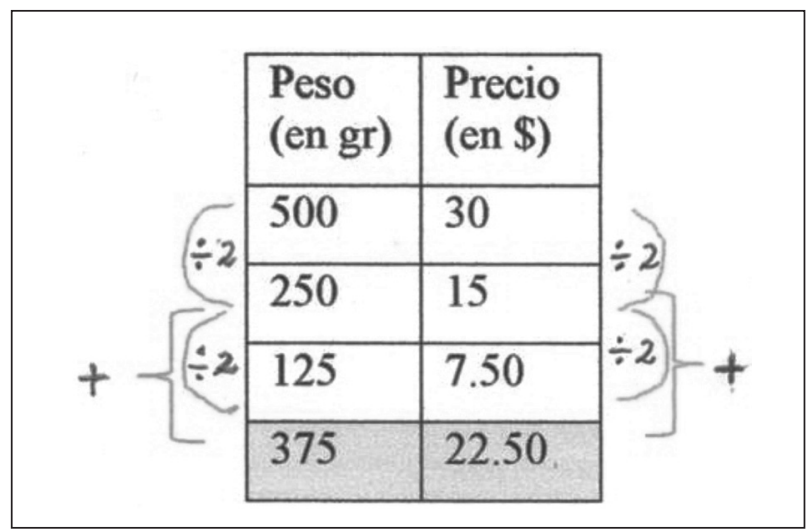

Figura 2 - Esquema de resolución mediante suma de mitades sucesivas de los valores conocidos

Esta estrategia, útil para calcular el costo de 375 gramos (o 750) en la situación planteada, se vuelve engorrosa al calcular ciertos valores (p.ej. el costo de 937 gramos), pues las composiciones necesarias para hacerlo son largas, cuestión que aumenta las exigencias de memoria y con ello dificulta la obtención de los resultados. Otra limitación de la estrategia es que al tener como punto de apoyo la obtención de mitades o duplicaciones sucesivas de los valores conocidos, no es posible obtener con exactitud algunas cantidades. En los problemas que nos ocupan, es claro que las duplicaciones y mitades sucesivas - y su composición - no conducirán a los precios exactos de 965 gramos, o al de 800, por lo que la estrategia deja de ser efectiva. Pero son pocas las personas que cuentan con otra para obtener la solución.

\subsection{Un uso incipiente del enfoque funcional: una unidad distinta de uno}

Sólo algunas personas han ideado una estrategia más efectiva para resolver estos problemas ${ }^{7}$. Es una estrategia que aproxima al valor de la unidad mediante un enfoque funcional (considerando la relación peso-precio), pero que luego regresa al uso de relaciones escalares para, así, obtener soluciones exactas o casi exactas en los dos casos expuestos (800 y 965 gr).

En esta estrategia, la unidad utilizada no es 1 - probablemente porque para calcular el valor de uno hace falta saber dividir - la unidad es100 gramos, o 50 gramos. Además, es posible subdividir la unidad y utilizar media unidad

\footnotetext{
${ }^{7}$ Dos estudiantes de primaria y una a secundaria.
} 
(p.ej. 25 gr habiendo tomado 50 gr como unidad) para obtener resultados exactos o más cercanos a éste.

Lo anterior puede considerarse un incipiente acercamiento al enfoque funcional, puesto que se establece la relación peso-precio entre 100gr (o 50 gr) y el costo correspondiente: $\$ 3$ (o $\$ 1.50$ ). Y este valor es la base para el resto de los cálculos que - como se observará en el ejemplo que se inserta adelante - se realizan de nuevo en el ámbito de las relaciones escalares.

Con esta forma de operar, y considerando 100 gramos como unidad (y $\$ 6$ como precio de esa unidad), el precio de 800 gramos puede obtenerse de la forma que se muestra en el esquema:

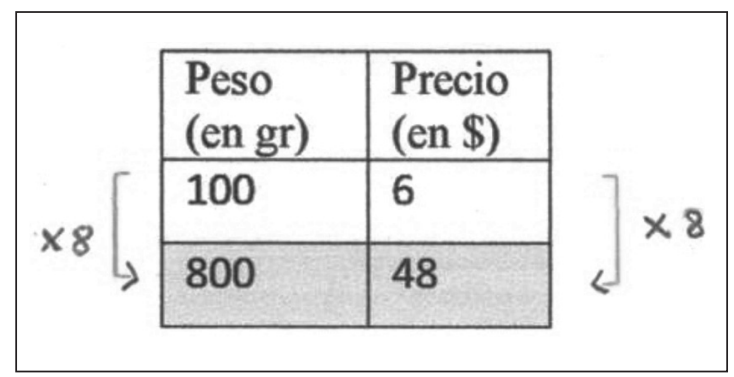

Figura 3 - Esquema de cálculo del precio de 800 gr, habiendo tomado 100 gr como unidad

Otro ejemplo del uso de esta estrategia, ahora habiendo tomado como unidad 50 gr, es el que se lee en el siguiente diálogo:

Entrevistador: El trozo de 800 gramos ¿cómo cuánto costaría?

Gerardo: ... a 3 pesos 50 gr... aquí [en 800 gramos] son 16 de 50 gramos... [entonces] le sumaría 16 por $3^{8} \ldots$

Entrevistador: ¿Cuánto te saldría? (Gerardo se queda pensativo, como calculando) Si quieres hacer la operación [en el papel]

Gerardo: (escribe):

$$
\begin{array}{r}
16 \\
\times 3 \\
\hline 48
\end{array}
$$

Gerardo: ... Serían 48 pesos por 800 gr.

\footnotetext{
${ }^{8}$ En realidad, Gerardo no suma, sino que hace, por isomorfismo, el cálculo correspondiente al precio, esta forma de expresión refleja seguramente sus limitaciones para verbalizar los cálculos que hace.
} 
La estrategia, basada en el manejo de una unidad diferente de uno, no conduce con exactitud el precio de 965 gramos, aunque permite una aproximación bastante buena: el precio de 975 gramos, aproximación que satisfizo a quienes la utilizaron.

Esta estrategia, aunque es bastante potente y flexible, no es una estrategia general que posibilite la obtención de resultados exactos en todos los casos (lo que se pedía era el precio de 965 gramos [\$57.90], no de 975: \$58.50). Es fácil percibir, también, que la memoria juega un rol importante para conservar en mente los cálculos parciales implicados. Es aquí donde es notoria la ausencia del conocimiento escolar. Es obvio que utilizarlo facilitaría notablemente los cálculos y, además, llevaría a obtener precios exactos. Pero, según se ve, las personas no recurren a él; ni la más ni las menos diestras con el cálculo oral. El escolar es un conocimiento que no ha impactado sus formas de hacer ni los alcances de estas formas.

\section{Estrategias utilizadas para resolver problemas de proporcionalidad: preeminencia de estrategias no escolares}

En virtud de lo hasta aquí expuesto, puede afirmarse que las formas de calcular que usan estas personas son, probablemente, las que utilizaban antes de asistir al servicio educativo. No se ve en su hacer la matemática escolar, la que debió haberse les comunicado en la educación básica. Se ve, en cambio, una matemática personal, resultado de enfrentar en la experiencia cotidiana - en mayor o menor medida - situaciones vinculadas a la proporcionalidad.

No realicé un análisis de las prácticas cotidianas que pudiesen implicar este tipo de situaciones, pero resultados de indagaciones previas y los derivados de ésta, permiten afirmar que estas estrategias son producto de la actividad cotidiana, principalmente la que se realiza durante la compra y venta de productos que se consumen con frecuencia, como los alimentos. Pero hay otra estrategia que se utilizó con destreza para resolver todos los problemas planteados. Es una estrategia que no tiene su origen en la escuela, sino que se construyó en el contexto laboral.

\section{Cuando el contexto comercial deviene contexto laboral, o el uso de la regla de tres situada}

Hasta aquí, los datos de nuestro estudio sobre la proporcionalidad no 
refieren a contextos laborales, sino al contexto escolar y al comercial. Sin embargo, este último contexto es también un espacio laboral para muchas personas, por ejemplo quienes tienen por ocupación vender (o cobrar) productos alimenticios, o de otro tipo, que deben ofrecerse a un precio proporcional al peso o la cantidad. Por este hecho, puede hacerse la hipótesis de que la actividad comercial - en tanto que actividad laboral - favorece la construcción y uso de estrategias de solución para problemas que implican proporcionalidad. Y así es, hay personas que mostraron contar con una estrategia útil para obtener todos los precios en la situación planteada durante la investigación que dio pie a este artículo. Son quienes utilizan la que he llamado regla de tres situada, que se desarrolla en el ámbito comercial y que comento a continuación.

Esquemáticamente, la estrategia consiste en lo siguiente: multiplicar el peso del producto solicitado (por ejemplo, $750 \mathrm{gr}$ ) el precio del kilo del mismo producto (por ejemplo \$50), para luego colocar el punto decimal en el lugar que los indicadores del contexto señalan como pertinente.

En el caso que se alude en esta tosca definición, tendríamos: 750 (peso del producto) $\times 50$ (costo del kilo del producto $)=37500 \rightarrow \$ 37.500$, donde el punto decimal no se coloca con base en una división, sino con base en el sentido común (sentido numérico) que se desarrolla mediante la experiencia. Los cálculos implicados en esta estrategia corresponden a los de la regla de tres convencional. Es una forma de operar que, muy probablemente, se trasmite al interior de la pequeña comunidad que constituye un puesto de ventas, o una tienda de abarrotes. Ignacio (uno de nuestros entrevistados) comentó que el dueño de la verdulería donde se contrató como empleado por un tiempo, le dijo al comenzar: Aquí se trabaja así: se multiplica el peso (del producto) por el precio (del kilo).

La estrategia - que siguiendo las palabras de Ignacio parece incompleta - se complementa con la colocación del punto (que resultaría de la división derivada de la regla de tres) en el lugar que el contexto del problema indica como razonable.

La regla de tres convencional llevaría a Ignacio a hacer, para calcular el costo de 800 gramos, los siguientes planteamientos y cálculos:

$$
\begin{aligned}
& \text { 1000: } 60:: 800: X \\
& 60 \times 800=48000 \\
& 48000 \div 1000=48.000
\end{aligned}
$$


Pero Ignacio tiene una importante destreza en el cálculo de precios que incluso le permite abreviar eliminando los ceros cuando esto es posible y conveniente:

$$
\begin{aligned}
& 800 \\
& \frac{X 6}{4800} \rightarrow 48.00
\end{aligned}
$$

Él lo explica así: Como [el producto] tiene el mismo precio [aunque se venda en distintas cantidades], se multiplica el 6, y el cero se pone ya al final, porque, es un decir, entre comillas, que el cero no tiene valor, es al final de cuentas donde se le ve.

Ignacio es un joven con una capacidad notable para resolver problemas de valor faltante, y, en general, problemas aritméticos. Pero, Ignacio no fue el único que utilizó esta estrategia. Aunque con menos agilidad, otras personas también la utilizaron, particularmente las dedicadas al comercio.

He llamado regla de tres situada a la estrategia recién descrita, porque su aprendizaje y uso tienen una amplia dependencia del contexto. De hecho, su aprendizaje deriva de y responde a las exigencias del ámbito en que se aplica cotidianamente: el comercio de frutas, verduras y otros productos que es necesario pesar o contar para cobrar correctamente (proporcionalmente) conforme al peso o la cantidad.

En la regla de tres situada se combina un procedimiento probablemente mecánico - no necesariamente se sabe por qué se multiplica y se divide lo que se multiplica y se divide - con el buen juicio de la experiencia cotidiana, que permite poner el punto decimal en el lugar correcto. Efectivamente, el lugar donde estas personas colocan el punto no deriva de una división, sino de otro factor: la experiencia matemática vital, que les permite ponderar el rango de validez de los resultados: Si el kilo vale 60 pesos, ;800 gramos no pueden valer 48000 , valen $\$ 48.00$ ! La habilidad para resolver de este modo se potencia con los registros escritos, como se ve en el caso de Ignacio, o con el uso de la calculadora, recurso que es bien apreciado por quienes se dedican a la actividad comercial.

Según nuestros datos, es en el contexto de la actividad comercial en 
donde se desarrolla este conocimiento, puesto que cuatro de las personas que utilizan la estrategia (a la quinta no se le preguntó) afirmaron trabajar o haber trabajado un tiempo (a veces largo) en una tienda de abarrotes o en una frutería. Se trata de una habilidad matemática situada, desarrollada en la actividad laboral, al interior de un gremio. Por supuesto, hace falta averiguar muchas más cosas respecto de este tema, sólo un ejemplo: si problemas de comparación de razones, o con la incógnita en el precio del kilo, o del peso del producto, se resuelven con la misma destreza que el tipo de problemas planteados, cuya pregunta refiere a lo que hay que cobrar.

\section{Las relaciones escuela-actividad cotidiana-trabajo en el desarrollo de otros conocimientos matemáticos}

\subsection{El cálculo de áreas en contexto de construcción}

Durante la investigación de la que deriva este reporte, planteamos un problema que involucra el área de un cuadrilátero irregular, en un contexto de construcción (Figura 4). En las respuestas de los participantes identificamos algo similar a lo observado con la proporcionalidad y su relación con el conocimiento escolar: las personas asistentes al servicio de educación básica se han apropiado de muy poco, o casi nada, del herramental que ese servicio educativo supone comunicar. En cambio, como resultado de la actividad laboral (de los albañiles, los pintores, los yeseros, los marmoleros) se observa el despliegue de destrezas y estrategias de solución que permiten resolver este tipo de problemas. En este ámbito, por ejemplo, se utiliza la estrategia que hemos llamado de los pintores, para calcular áreas de ciertas superficies (DE AGÜERO, 2006; ESTRADA; ÁVILA, 2009). 
Don Antonio va a pintar una pared como la que aparece en el dibujo.

Observe las medidas y responda las siguientes preguntas:

¿Cuántos metros cuadrados debe pintar don Antonio?

Si don Antonio cobra a \$ 30.00 el metro cuadrado, incluyendo la pintura, ¿cuánto le pagarán por pintar la pared?

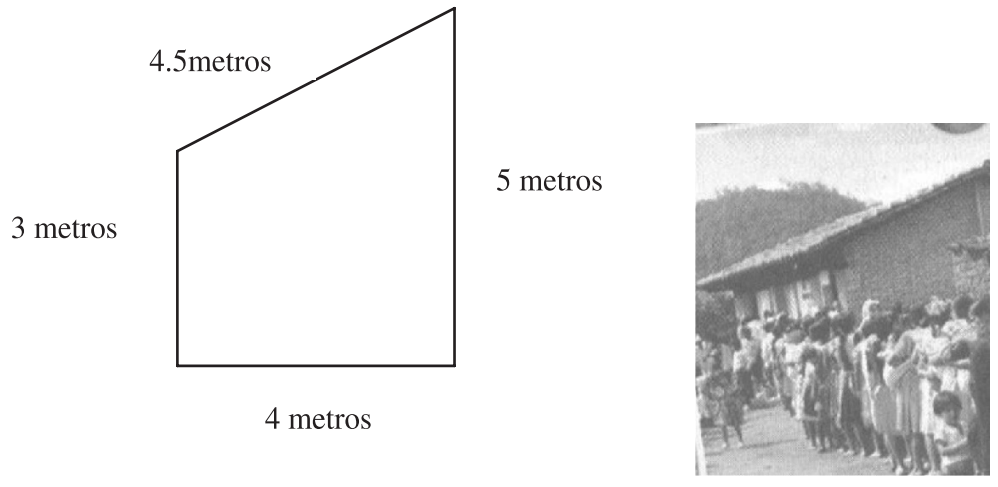

Figura 4 - Situación planteada que implica la resolución de un problema de área

La estrategia de los pintores hace evidente la flexibilidad y agudeza en el manejo del espacio de las personas dedicadas a actividades relacionadas con la construcción, y también la posesión de estrategias que parecen compartirse en el gremio. En el problema planteado, el cálculo del área se obtuvo mediante multiplicación de la medida de la base de la pared por la altura media de la misma $(4 \times 4,5)$, lo que da por resultado un área de $18 \mathrm{~m}^{2}$ (véase Figura 5). ${ }^{9}$

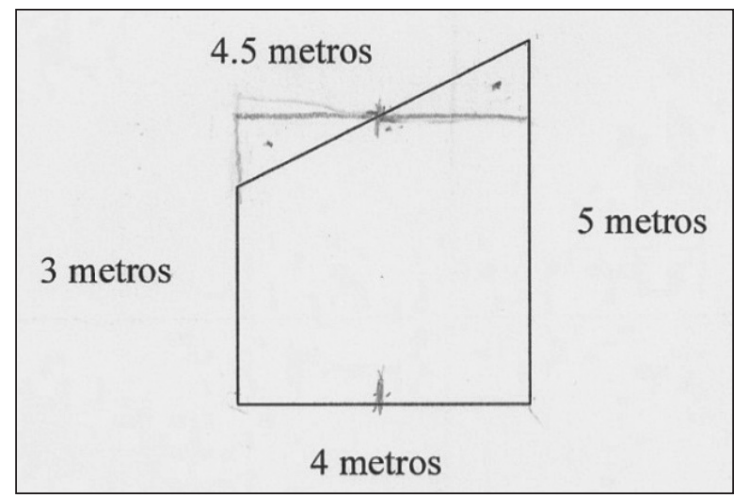

Figura 5 - Dibujo que apoya la estrategia de solución de Sergio, estudiante de primaria y dedicado al oficio de yesero durante 11 años

${ }_{9}^{9}$ La validez de este procedimiento se expone en Estrada y Avila (2009). 
Esta estrategia - identificada inicialmente por De Agüero (2006) y después por Estrada y Ávila (2009) - fue movilizada por nuestros entrevistados dedicados a alguna de las actividades vinculadas a la construcción. Y a excepción de una mujer, asistente a secundaria, que hizo una aproximación razonable mediante un procedimiento escolar, el resto de las personas no desplegaron estrategias pertinentes para resolver el problema.

\subsection{El cálculo aritmético en contextos de compra-venta}

Datos ya viejos, recogidos hace 20 años en mis primeros acercamientos a este tema (AVILA, 1989), muestran también que, en el cálculo aritmético, puede tener incidencia el trabajo que las personas realizan. Según nos contó en ese entonces un inteligente analfabeto que hacía funciones de mozo en la casa de una familia acomodada, y cuya destreza en el cálculo era notable, su aprendizaje sobre las cuentas se debió a las exigencias de su patrona, quien durante 20 años lo mandó al mercado a hacer las compras:

De regreso de las compras, en que presentaba el ticket [la hoja con los encargos] que la señora me había apuntado, me fijaba yo lo que me había costado y, por el camino, ya cuando venía en la calle, venía haciendo las cuentas así, con la boca y con los dedos... lo normal era que se me grababa: las zanahorias me costaron a tanto el kilo, las calabazas a tanto [...] y ahí es donde va uno agarrando las cosas, las ideas [...] (AVILA, 1989, p. 148).

Pero no siempre la actividad que se realiza en el contexto comerciolaboral corre a favor de las personas, en el sentido de promover el desarrollo de sus conocimientos y destrezas matemáticas. Tal es el caso de Delfina, una mujer analfabeta que no elevaba los precios de sus productos - así obtuviera pocas ganancias - porque los que había fijado hacía tiempo correspondían a las cuentas que sabía hacer (AVILA, 1989). Esta mujer, cuando le preguntamos cómo hacía entonces para comprar el pan, respondió: "Pues compro el pan, y no más digo, ¿cuánto es lo del pan?, entonces lo pago y me dan el cambio, y no sé ni cuánto es [...] luego mejor mando a mi chamaca" (AVILA, 1989, p. 145).

Se ve, en el caso de Delfina - al igual que en el de quienes no pudieron resolver los problemas de planteados durante esta investigación - que los conocimientos desarrollados en la vida pueden ser muy escasos. Sería altamente deseable que la escuela proporcionara a estas personas las condiciones necesarias para desarrollar las destrezas que su contexto natural no promovió. 


\section{Discusión}

En este escrito analicé los aportes de la educación básica, la experiencia de vida y la actividad laboral a la alfabetización matemática de las personas, es decir, a su capacidad de identificar información cuantitativa, valorarla críticamente, reflexionar sobre ella y a partir de ella, y aplicarla al enfrentar actividades y problemas del ámbito social, laboral y personal. Lo hice, ciertamente, centrada en el primero de estos ámbitos, pero el progreso en la investigación que dio pie a este escrito me condujo a ampliar la perspectiva inicial y a considerar otras influencias en el hacer matemático de las personas.

En relación con la proporcionalidad, constaté que no se utilizan los procedimientos propios de la matemática escolar, ni aun cuando la determinación de precios exactos hace indispensable dicho uso. Es cierto que la situación sugerida durante la indagación era ficticia y las personas sabían que no perderían dinero (motivación poderosa para la realización de buenos cálculos y motor del desarrollo de la capacidad de calcular). Pero el supuesto de partida era que la situación haría aflorar los conocimientos, producto de experiencias previas, fueran éstas experiencias de vida o escolares. Sin embargo, entre estos conocimientos no se observan los procedimientos convencionales escritos que facilitarían enormemente la obtención de las soluciones, sino solamente aquéllos que cada uno parece haber construido en su hacer matemático vital.

En otro trabajo (AVILA, 2007) afirmé que la contribución de la escuela a la alfabetización matemática debiera ser aportar las simbolizaciones y procedimientos escritos generales, de manera que su adquisición potenciara la capacidad de resolver problemas y, vinculado con esto, las formas de resolución utilizadas cotidianamente. Dicha simbolización y dichos procedimientos, de ser adquiridos significativamente, permitirían resolver todos los casos, más allá de los específicos implicados en el contexto en que las personas realizan su actividad matemática cotidiana.

Este estudio puso en evidencia que, en el caso de la proporcionalidad, tales expectativas no se cumplen, y aunque los saberes de la experiencia encuentran su límite en los problemas donde es necesario utilizar los procedimientos escolares para obtener resultados exactos, la pérdida de eficacia en el cálculo no lleva a movilizar estrategias escritas generales que permitirían alcanzar cualquier resultado en un número finito de pasos.

Con este y otros ejemplos, como el de la capacidad de resolver ciertos problemas aritméticos o de cálculo de áreas, creo haber mostrado que las 
actividades laboral y comercial son fuentes importantes de conocimientos necesarios para responder a las exigencias del trabajo y del intercambio comercial que realizan cotidianamente las personas. En cambio, los resultados de la acción escolar son poco visibles.

Sabemos, por otra parte, que la matemática que se construye en ámbito laboral, siendo funcional, tiene límites, porque los procedimientos que ahí se construyen no tienen la potencia de los generales que la escuela debiera trasmitir. ¿Cómo podría la educación formal lograr la complementariedad necesaria entre estos dos saberes?, ¿La complementariedad sería posible y deseable respecto de todos los contenidos matemáticos escolares? Las respuestas obligan aún a la indagación, pero la cuestión no es simple.

Trabajos como el de Boero (2009) constatan que las relaciones entre la matemática que se enseña en la escuela y la que se aprende en la vida son complejas ya desde la infancia, y que hay una gran proclividad a trivializarlas. Más complejas se vuelven estas relaciones cuando no se trata de niños, sino de personas que han elaborado un sistema de cálculo aritmético - o de medición a partir del cual se vinculan con la matemática escolar. En este caso, tal vez resulte más fácil trivializar los vínculos (MARIÑO, 1997).

En el mismo sentido, y en un trabajo reciente, Solares (2011) alerta sobre el hecho de que no siempre parece posible establecer relaciones directas entre los conocimientos matemáticos escolares y los no escolares. Lo hace a partir de una situación de medición en un contexto agrícola. Comparto plenamente los llamados a la prudencia. En cambio, no comparto (al menos no por ahora) la opinión de quienes consideran innecesario (o imposible) el vínculo entre el saber matemático de la vida y el de la escuela. Tal postura se ha construido bajo la creencia de que el conocimiento de la vida ahí está, y las personas no necesitan ir a la escuela para adquirirlo. En contraparte, dicen, la matemática que se estudia en la escuela (de jóvenes o adultos en rezago) es difícilmente transferible a la vida y sus objetivos son de otro tipo: es útil para seguir estudiando y para obtener certificaciones de utilidad pragmática, no para potenciar la actividad matemática cotidiana. Esta postura, extrema, llevaría a decisiones a priori. Considero que el trabajo y los intercambios comerciales son una fuente importantísima de conocimientos matemáticos, pero la escuela - también la de jóvenes y adultos debe hacer su parte y proporcionar aquellos conocimientos matemáticos que no da la vida, pero que también son útiles en la vida.

De tal necesidad son muestra las complicadas soluciones utilizadas por los participantes en esta investigación porque - parafraseando a Guy Brousseau 
(1978) - los saberes también toman sentido (y son útiles) por las economías que procuran.

Desde una perspectiva distinta, los saberes prácticos y simbólicos reportados por De Agüero (2006) en su trabajo sobre una cuadrilla de pintores, también constatan la relevancia de que el saber escolar permee y complemente los saberes no escolares. La pequeña comunidad de práctica constituida por los pintores, sobrevive y tiene éxito porque - además de los saberes matemáticos prácticos que se han desarrollado en su interior - un miembro de la comunidad posee el herramental simbólico que sus años de escolaridad le proporcionó, y de esa posesión depende una vinculación más o menos horizontal con el exterior.

¿Cómo hacer para lograr esta complementariedad?, sólo tengo ideas sueltas, pero ninguna de ellas escinde el saber de la escuela y el de la vida. Tengo también una convicción, ya expresada por otros investigadores (EVANS, 2002; VERGANAUD, 2002), acerca de que la alfabetización matemática plena es el resultado de diferentes procesos: la educación escolarizada, la experiencia de vida y la formación en el trabajo. No podemos menospreciar o sobrevalorar uno u otros, ni confundir la mala calidad de la educación matemática escolar que se ofrece a los adultos con su irrelevancia.

Es obvio que las escuelas de educación básica para adultos deben cambiar. La no integración - en el hacer matemático de las personas - de lo que ahí se busca comunicar, refleja la precariedad del servicio que se ofrece. Pero más allá de ello, quedan largos trechos por recorrer para comprender las relaciones pertinentes que los educadores hemos de establecer entre los tres ámbitos abordados en este escrito, de manera de contribuir a la alfabetización matemática de las personas, en el sentido más amplio de la frase. Una vez dilucidado el carácter de estos vínculos, podremos repensar, con mejores herramientas, la educación matemática escolar de los jóvenes y adultos y, si es necesario, reconstituirla.

\section{Referencias}

AVILA, A. Estrategias e cálculo aritmético de los adultos no alfabetizados. 1989. 162 f. Tesis (Maestría en Pedagogía) - Facultad de Filosofía y Letras, Universidad Nacional Autónoma de México, México DF, 1989.

AVILA, A. El saber matemático de los analfabetos. Origen y desarrollo de sus estrategias de cálculo. Revista Latinoamericana de Estudios Educativos, México, v. 20, n. 3, p. 55-95, 1990. 
AVILA, A. Del cálculo oral al cálculo escrito. Una batalla para acceder a una nueva significación. Recherches en didactique des mathématiques, Grenoble, v. 27, n. 3, p. 313-347, 2007.

AVILA, A. ¿Del cálculo oral al cálculo escrito? Constataciones a partir de una situación de proporcionalidad. En: KALMAN, J.; STREET, B. V. (Coords.). Lectura, escritura y matemáticas como prácticas sociales. México: CREFAL-Siglo XXI, 2009. p. 223-241. (Diálogos con América Latina).

AVILA, A. et al. Las matemáticas en la educación indígena. Análisis de sus condiciones y problemas. México: Universidad Pedagógica Nacional - Dirección General de Educación Indígena. Reporte de investigación impreso no publicado, 2010.

AVILA, A. et al. Matemáticas y educación de jóvenes y adultos. Estudio a través de la voz y el saber de los usuarios. México. Universidad Pedagógica Nacional/Universidad Autónoma de Aguascalientes/Instituto de Educación de Aguascalientes. Reporte de investigación impreso no publicado, 2008.

BOERO, P. Les domaines d'expérience dans l'enseignement - apprentissage des mathématiques: lier le travail scolaire à l'expérience de l'élèves. En: MARGOLINAS, C . et al. (Coords.). En Amont et en Aval des Ingenieries Didactiques: $15^{\mathrm{e}}$ Ecole d'été de Didactique des Mathématiques. Francia: La Pensée Sauvage Éditions, 2009. p. 111148.

BROUSSEAU, G. Les obstacles épistémologiques et les problèmes en mathématiques. Recherches en didactique des mathématiques, Grenoble, v. 4, n. 2, p. 165-198, 1978.

CARRAHER, T.; CARRAHER, D.; SCHLIEMANN, A. En la vida diez, en la escuela cero. México: Siglo XXI Editores, 1991.

DE AGÜERO, M. El pensamiento práctico de una cuadrilla de pintores. México: CREFAL/Universidad Iberoamericana, 2006.

ESTRADA, J. L.; ÁVILA, A. Los usuarios de la Educación Básica para Jóvenes y Adultos y la resolución de un problema de área. Educación Matemática, México, v. 21, n. 3, p. 33-66, dic. 2009.

EUDAVE, D. Invitación al Coloquio Alfabetización Académica, Informativa y Numérico-estadística en universitarios. México, Universidad Autónoma de Aguascalientes, 13-14 nov. 2010.

EUDAVE, D. Niveles de comprensión de información y gráficas estadísticas en estudiantes de centros de educación Básica para jóvenes y adultos de México. Educación Matemática, México, v. 21, n. 2, p. 5-38, ago. 2009. 
EVANS, J. The transfer of mathematics learning from school to work, not straight forward but not impossible neither! En: BESSOT, A.; RIDWAY, J. (Orgs.). Education for Mathematics in the workplace. Kluwer: Academic Publishers, 2002. p. 5-16.

FERNÁNDEZ, C.; LLINARES, S.; VALLS, J. Características del desarrollo de una mirada profesional en estudiantes para profesor de matemáticas en un contexto blearning. Acta Scientiae, Canoas, v. 13, n. 1, p. 9-30, jan./jun. 2011.

GAL, I. Issues and Challenges in Adult Numeracy. En: MEMORIA DEL SEMINAIRE INTERNATIONALL'ENSEIGNEMENTETL'APPRENTISAGEDES MATHEMATIQUES DE BASE AUX JEUNES ET AUX ADULTES DEFAIBLE NIVEAU, 1., 1993, Marly Le Roi. Anales... Angers: UNESCO/Centre Universitaire de Formation Continue. Université d'Angers, 1993. p. 114-141.

MARIÑO, G. Cómo opera matemáticamente el adulto del sector popular? Constataciones y propuestas. Bogotá: Dimensión Educativa, 1983.

MARIÑO, G. Los saberes matemáticos previos de jóvenes y adultos: alcances y desafíos. En: LIZARZABURU, A. (Org.). Conocimiento matemático en la educación de jóvenes y adultos. Santiago de Chile: UNESCO, 1997. p. 77-100.

SOLARES, D. Conocimientos matemáticos en situaciones extraescolares. Análisis de un caso en el contexto de los niños y niñas jornaleros migrantes. Educación Matemática, México, v. 24, n. 1, p. 5-33, abr. 2011.

SOTO, I.; ROUCHE, N. Problemas de proporcionalidad resueltos por campesinos chilenos. Educación Matemática, México, v. 7, n. 1, p. 77-95, abr. 1995.

VERGNAUD, G. Introduction. En: BESSOT, A.; RIDWAY, J. (Orgs.). Education for Mathematics in the workplace. Kluwer: Academic Publishers, 2002. p. XVII - XIV.

Submetido em Outubro de 2012. Aprovado em Fevereiro de 2013. 
Anexo 1 - Porcentajes de tipos de respuestas, por nivel educativo, a los problemas de proporcionalidad planteados durante la investigación

\begin{tabular}{|c|c|c|c|c|c|c|c|c|c|c|c|c|c|c|c|c|c|c|}
\hline & \multicolumn{6}{|c|}{ Exactas } & \multicolumn{6}{|c|}{ Aproximadas } & \multicolumn{6}{|c|}{ Erróneas o no proporcionadas } \\
\hline & $\begin{array}{l}25 \\
0 \\
\mathrm{gr}\end{array}$ & $1 \mathrm{~kg}$ & $\begin{array}{l}50 \\
\mathrm{gr}\end{array}$ & $\begin{array}{l}375 \\
\mathrm{gr}\end{array}$ & $\begin{array}{l}800 \\
\mathrm{gr}\end{array}$ & $\begin{array}{l}965 \\
\text { gr }\end{array}$ & $\begin{array}{l}25 \\
0\end{array}$ & $\begin{array}{l}1 \\
k \\
\mathrm{~g}\end{array}$ & 50 & 375 & 800 & 965 & $\begin{array}{l}250 \\
\mathrm{gr}\end{array}$ & $1 \mathrm{~kg}$ & 50 & 375 & 800 & 965 \\
\hline Sec. & 94 & 88 & 37 & 25 & 19 & 19 & 6 & $\begin{array}{l}0 \\
2\end{array}$ & 44 & 44 & 56 & 50 & 0 & 0 & 19 & 31 & 25 & 31 \\
\hline Prim & 70 & 70 & 40 & 40 & 40 & 20 & 0 & 0 & 20 & 30 & 30 & 40 & 30 & 30 & 30 & 30 & 30 & 40 \\
\hline $\begin{array}{l}\text { Totales } \\
\text { por } \\
\text { ítem }\end{array}$ & 85 & 81 & 38 & 31 & 27 & 19 & 23 & 8 & 35 & 38 & 46 & 46 & 12 & 12 & 23 & 31 & 27 & 35 \\
\hline $\begin{array}{l}\text { Totales } \\
\text { en el } \\
\text { conjunt } \\
\text { o de } \\
\text { respues } \\
\text { tas }\end{array}$ & \multicolumn{6}{|c|}{$46.8 \%$} & \multicolumn{6}{|c|}{$32 \%$} & \multicolumn{6}{|c|}{$23.3 \%$} \\
\hline
\end{tabular}

Primaria: $\mathrm{N}=10$ Secundaria: $\mathrm{N}=16$ 